\title{
Active impact on the ionosphere and variations of velocity char- acteristics of field-aligned irregularities
}

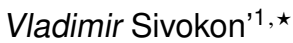 \\ ${ }^{1}$ Institute of Cosmophysical Research and Radio Wave Propagation FEB RAS, Kamchatskiy kray, Paratunka, \\ Russia
}

\begin{abstract}
Ionospheric irregularities make significant impact on the efficiency of radioengineering system operation. Our investigations carried out on Kamchatka peninsula showed that field-aligned irregularities have particularly destructive influence on decameter radars. We pin some definite expectations with them to increase the efficiency of radioengineering systems by active impact on the ionosphere. One of the important parameters of such irregularities is their velocity characteristics. When determining irregularity velocity in the impact area, different approaches are used, for example, aspect scattering method which specifies velocity on the whole but does not allow one to determine its vertical and horizontal components, direction of its motion. We have developed an approach which allows us to solve this problem. As an example, results of observations over EISCAT experiments in October 2019 are presented.
\end{abstract}

\section{Introduction}

Our investigations [1] showed that presence of a special class of ionospheric irregularities, fieldaligned ones, is acute not only for beyond-the-horizon radio location. This type of irregularities is of interest during active impact on the ionosphere [2]. Consequently, research of field-aligned irregularities is a topical task.

In order to estimate the parameters of artificial irregularities of the ionosphere, different methods are applied, for example, test wave [3], aspect [4] and backward scattering [5] methods. To realize these methods one needs complex equipment unpurchasable for the majority of researches. We suggested a method [6] which included generally acceptable software-defined SDR receivers (SoftDefinedRadio). Results of the observation over the experiments taking place in October 2019 are described in the paper.

\section{Method justification}

A network of SDR receivers installed in Europe, in particular, make it possible to record electromagnetic radiation from the impact area within the line-of-sight, that minimizes the ionosphere impact

\footnotetext{
${ }^{\star}$ Corresponding author: nina@ikir.ru

The paper was carried out within the framework on the subject «Dynamics of physical processes in active zones of near space and geospheres» (AAAA-A17-117080110043-4)
} 
on radiation propagation. An analysis of the distribution of the amplitudes of the heating radiation scattered by ionospheric irregularities showed it's bimodal and, in some cases, polymodal character. When estimating the capacity of the short-wave communication channel (ionosphere heating is carried out just in this wave length range) one take Rayleigh, Rice or Nakahami distributions [7], as a rule. However, they are not bimodal, i.e. the observed distributions are anomalous with respect to those distributions. Based on the velocities of the observed fading, Fig.1, they refer to fast ones.

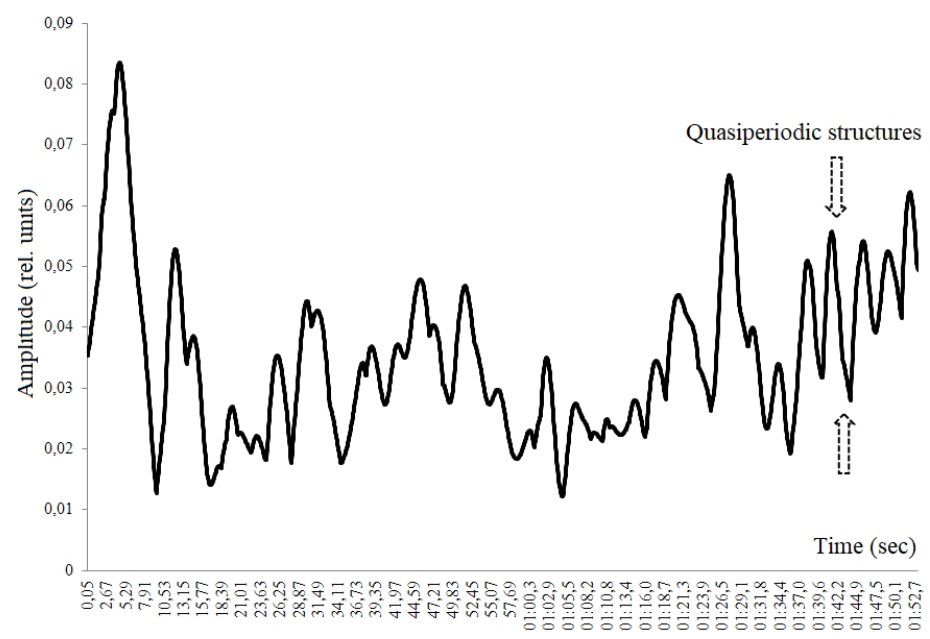

Figure 1. Scattered signal record on October 18, 2019 at 13:14 UT at Alta

Fast fading is divided into interference and polarization ones. It is known that polarization fading is determined by magneto-ionic splitting of the wave incident to the ionosphere and, in a general case, by the formation of elliptically polarization signal the polarization characteristics of which do not coincide with the same characteristics of a receiving antenna. However, under active impact on the ionosphere, when the direction is towards the magnetic zenith, radiation with circular polarization is used and the possibility of polarization fading manifestation is low as long as in this case there will always be a position of electric field strength vector which will coincide with receiving antenna polarization plane $[8,9]$. Interference fading is, as a rule, determined by ray path differences, i.e., phase ratios. In our case, it is necessary to take into account frequency Doppler shift as long as fieldaligned irregularities move [1]. Then, in a general case, the resultant field can be represented as a sum

$$
E(t)=E_{1} \cdot \cos \left(\omega_{1} t+\varphi_{1}\right)+E_{2} \cdot \cos \left(\omega_{2} t+\varphi_{2}\right)+\ldots+\xi(t),
$$

where $E_{1}, E_{2}$ are amplitudes; $\omega_{1}, \omega_{2}$ are frequencies; $\varphi_{1}, \varphi_{2}$ are phases of separate scattered components; $\xi(t)$ is the noise component.

For the case when $\omega_{1}, \omega_{2}$ differ insignificantly and noise dispersion is small, in the paper [10] an approximated expression for the density of process envelope possibility for two-ray distribution was obtained

$$
\begin{gathered}
W(R) \cong \frac{R}{12 \sigma^{2}} \exp \left[-\frac{1}{2 \sigma^{2}}\left(R^{2}+E_{1}^{2}+E_{2}^{2}\right)\right] \times\left\{P I_{0}\left[\frac{E_{2}}{\sigma^{2}}\left(R-E_{1}\right)\right]+\frac{1}{P} I_{0}\left[\frac{E_{2}}{\sigma^{2}}\left(R+E_{1}\right)\right]+\right. \\
\left.+2\left[P I_{0}\left(\frac{E_{2}}{\sigma^{2}} \sqrt{R^{2}-R E_{1}+E_{1}^{2}}\right)+\frac{1}{P} I_{0}\left(\frac{E_{2}}{\sigma^{2}} \sqrt{R^{2}+R E_{1}+E_{1}^{2}}\right)\right]\right\}
\end{gathered}
$$


where $P=\exp \left(-\frac{R E_{1}}{2 \sigma^{2}}\right)$.

When choosing the parameters $E_{1}, E_{2}$ and $\sigma$ we can obtain bimodal distribution and component correlation is not necessary in this case. The main requirement is the difference of frequencies $\omega_{1}$ and $\omega_{2}$. As long as this difference is determined by frequency Doppler shift, it cannot be significant compared to the heating frequency, then quasi-beats and quasi-periodic processes should take place. In Fig. 1, features of such a process are indicated by arrows.

\section{Observation results}

Besides the features of beats in a wave form, we detected spectrum splitting, Fig.2. This phenomenon is described in the papers $[10,11]$. In the paper [10] it is assumed that this phenomenon is determined by field-aligned irregularity drift.

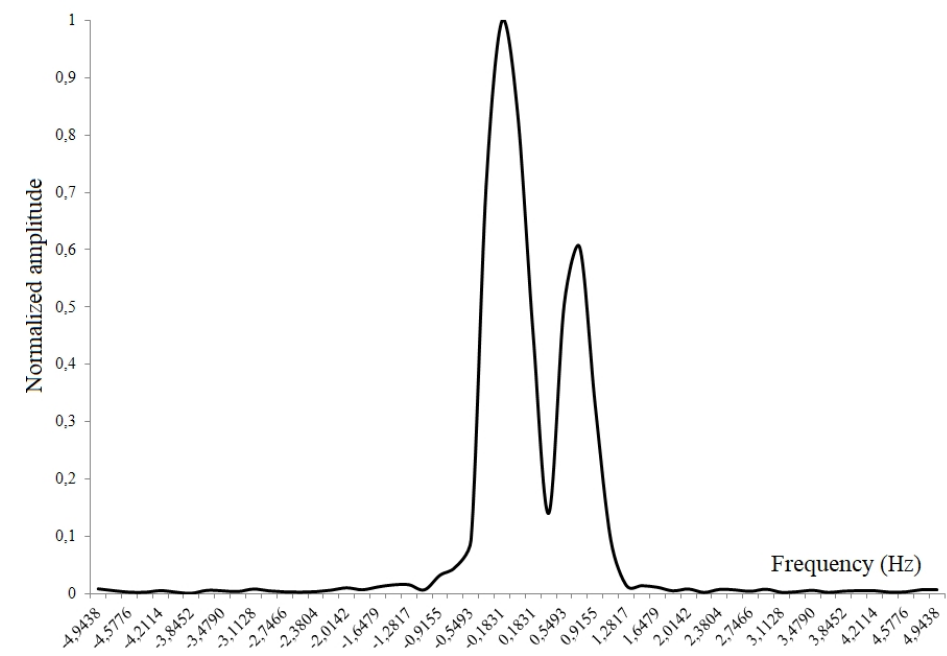

Figure 2. Scattered signal record on October 18, 2019 at 13:14 UT at Alta

Spectrum splitting is an interesting phenomenon but during automatic processing of records it causes difficulties in detection of real value of frequency Doppler shift. As long as at the current stage of investigations the experimental data volume is not large and our resources are limited, we made some simplification, the software determines frequency Doppler shift for the maximum amplitude.

At the initial stage of investigations when estimating velocity and direction of irregularity motion, we applied ten-second averaging that is, probably, unjustified for the irregularity parameters described above. Thus, when we processed the data from October 2019, another approach was used. In software we defined the FFT maximum and the maximum time resolution.

Based on the initial data, frequency Doppler shift distributions were drawn and velocity distributions were calculated from them. As long as it is not possible to present the data over the whole heating cycle, we divided the cycle into periods of power increase and decrease. Results for ALTA site are illustrated in Fig.3-4. Markers show velocity discrete values, the lines connecting the markers are applied in order to trace easier velocity changes at the heating stage. It is clear from the graphs that when the impact power increases, the velocity value and sign change. Under minimum impact, the irregularities moved away from an observer. As the power grew, they began moved in an opposite 


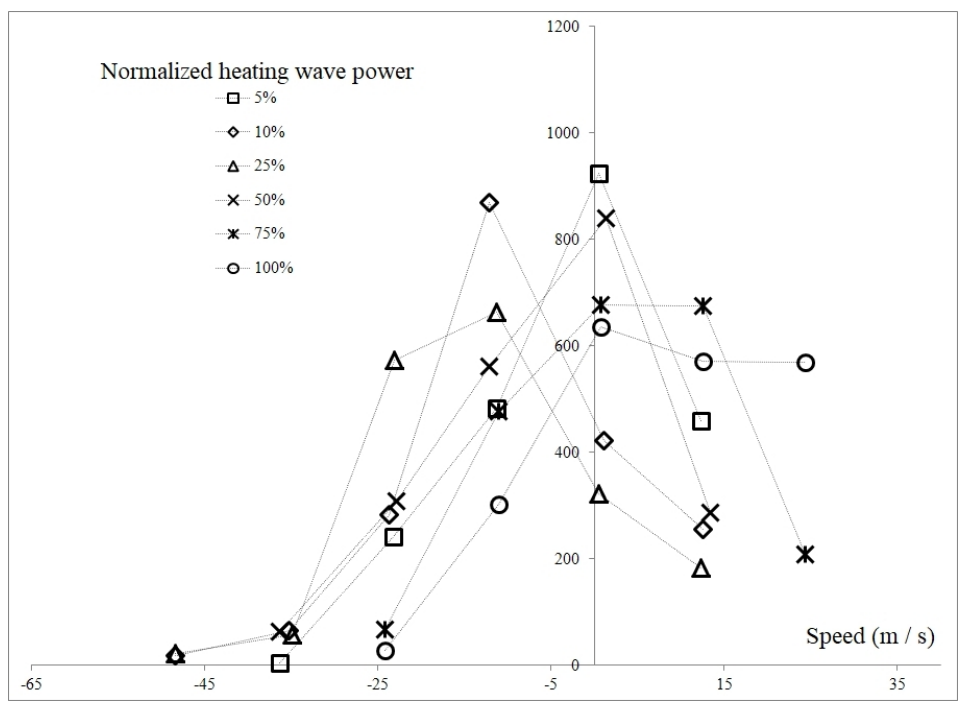

Figure 3. Velocity distribution during impact power increase

direction. When impact power decreased, we expected an opposite picture, that occurred, however, the process was slow that may indicate the inertial process in ionospheric plasma.

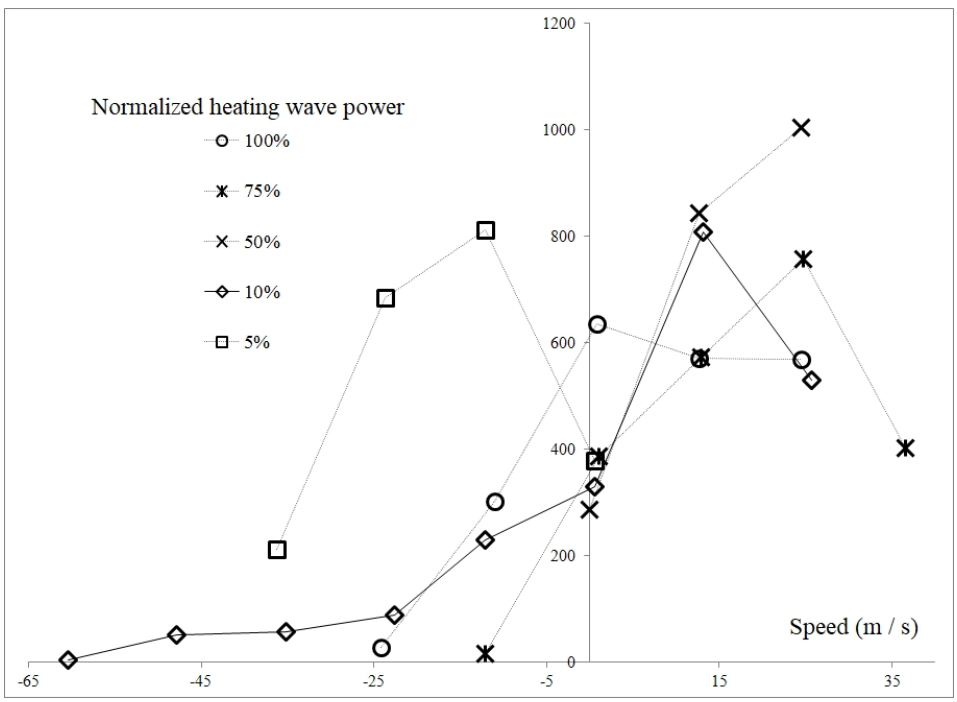

Figure 4. Velocity distribution during impact power decrease

We use multi-position diagnosis of ionosphere modification, i.e. the area is observed at different angles in vertical and horizontal planes, that allows us to determine the components of irregularity velocity and direction of their primary motion, Fig.5. 


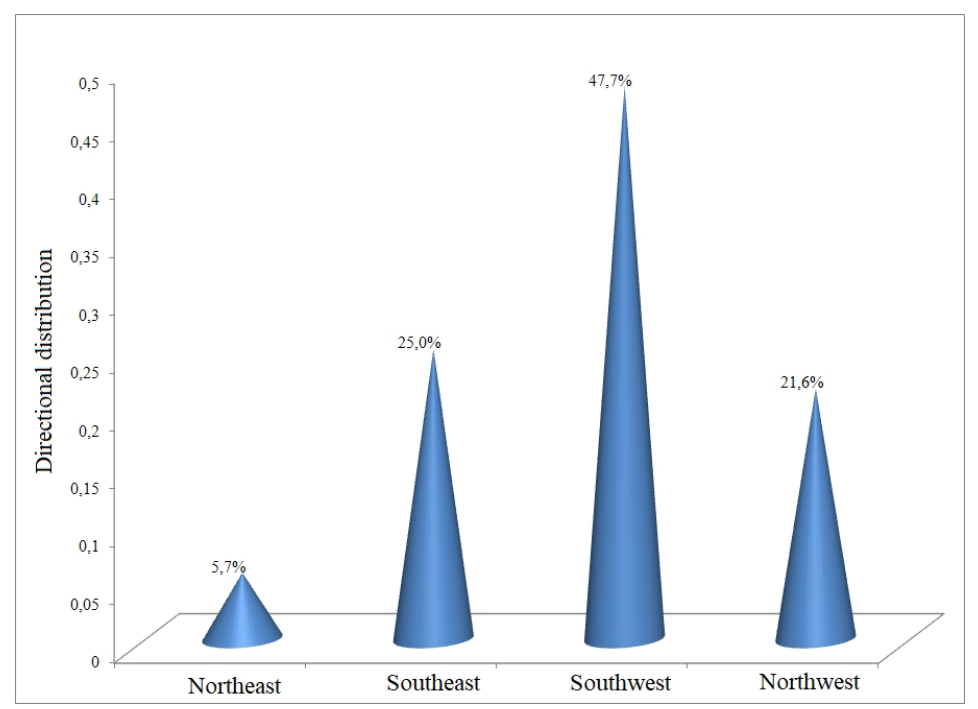

Figure 5. Distribution of irregularity motion directions

\section{Conclusions}

- We have shown the possibility to investigate velocities and directions of ionospheric field-aligned irregularity motion applying SDR technology.

- Observations showed the dependence of motion velocity and direction on heating wave power.

- The motion determined by active impact on the ionosphere has primary southern direction.

\section{References}

[1] V.P. Sivokon, Geomagnetism and Aeronomy, 57(3), 317-325 (2017) DOI: 10.1134/S001679321702013X

[2] N.F. Blagoveshchenskaya, T.D. Borisova, A.S. Kalishin, T.K. Yeoman, Artificial Small-scale Field-aligned Irregularities in the High Latitude Ionospheric F-region, AGU Fall Meeting 10 14 December (Washington, DC, USA, 2018)

[3] S.M. Grach, G.P. Kompakov, M.M. Shvarts, M.A. Yurishchev, Radiophysics and Quantum Electronics, 41(8), 653-660 (1998) DOI: 10.1007/BF02676473

[4] N.F. Blagoveshchenskaya, T.D. Borisova, V.A. Kornienko, T.R. Robinzon,T.K. Yoman, V.L. Frolov, M.T Ritvel'd, Solnechno-zemnaya fizika [Solar-Terrestrial Physics], 12(2), 206-209 (2008) (in Russian)

[5] N. A. Mityakov, V. A. Alimov, V. A. Zinichev, G. P. Komrakov, S. N. Mityakov, Radiophysics and Quantum Electronics, 53(5-6), 297-304 (2010) DOI: 10.1007/s11141-010-9228-6

[6] V.P. Sivokon', R.V. Kolesnikov, I.V. Demichev, Elektromagnitnye volny i elektronnye sistemy [Electromagnetic waves and electronic systems], 24(8), 23-28 (2019) (in Russian) DOI: 10.18127/j15604128-201908-03

[7] D.D. Klovskiy Peredacha diskretnykh soobshcheniy po radiokanalam [Transmission of discrete messages via radio channels], (Moscow: Radio i svyaz', 1982), -304 p. (in Russian) 
[8] V.P. Sivokon', Elektrosvyaz' [Telecommunications and Radio Engineering], 7, 55-58 (2007) (in Russian)

[9] V.P. Sivokon, G.I. Druzhin, Geomagnetism and Aeronomy, 46(4), 492-495 (2006) DOI: 10.1134/S0016793206040116

[10] A.V. Koloskov, V.S. Beley, T.B. Leyzer, Yu.M. Yampol'skiy, Radiophysics and Radioastronomy, 4(3), 247-260 (1999) (in Russian) http://rpra-journal.org.ua/index.php/ra/article/view/978

[11] T.D. Borisova, N.F. Blagoveshchenskaya, V.A. Kornienko, V.L. Frolov, V.V. Vertogradov, V.G. Vertogradov, Geomagnetism and Aeronomy, 49(4), 535-544 (2009) (in Russian) 